\title{
La religion dans Malos Tiempos de Juan Madrid
}

\author{
The Religion in Malos Timpos of Juan Madrid
}

\author{
Maguette Dieng* \\ maguette3819@yahoo.fr \\ Université Cheikh Anta Diop de Dakar
}

RÉSUMÉ : Investissant le Res factae, l'auteur espagnol de roman noir Juan Madrid réunit dans Malos tiempos (2017) les récits de huit faits divers ensanglantés qu'il avait déjà publiés en tant que reporter dans Cambio 16. Les meurtres qu'on y relate dérivent tous des attributs de la société contemporaine: le matérialisme, la cupidité, l'individualisme etc. La religion, en tant qu'instrument d'aliénation et sa marchandisation, est à l'origine de l'horrible crime commis par les Hermanas de luz, adeptes de la Sainte Lucie. Cet article se veut une étude basée sur une approche d'ordre herméneutique, conciliant la perspective théorico-conceptuelle et épistémologique avec la pratique textuelle.

MOTS-CLEFS : Religion. Aliénation. Marchandisation. Reportage. Déshumanisation.

RESUMO: Investindo a Res factae, o autor espanhol do romance negro Juan Madrid reúne em Malos tiempos (2017) as histórias de oito eventos sangrentos que já havia publicado no Cambio 16 enquanto repórter. Os assassinatos nelas relatados derivam de atributos da sociedade contemporânea: materialismo, ganância, individualismo e assim por diante. A religião, como instrumento de alienação e de sua mercantilização, está na origem do crime horrível cometido por Hermanas de Luz, seguidoras de Santa Lúcia. Este artigo é um estudo baseado em uma abordagem hermenêutica, conciliando a perspectiva teórico-conceitual e epistemológica com a prática textual.

PALAVRAS-CHAVE: Religião. Alienação. Mercantilização. Reportagem. Desumanização.

ABSTRACT: Investing the Res factae, the Spanish author of black novels Juan Madrid gathers in Malos tiempos (2017) the stories of eight bloody local news that he had already published as a reporter in Cambio 16 . The murders reported there derive

\footnotetext{
* Chef du Bureau animation et organisation pédagogique de la Direction des Affaires Pédagogiques (DAP); Chef du département de Langues Romanes de la Faculté des Sciences et Technologies de l'Éducation et de la Formation (FASTEF) de l'Université Cheikh Anta Diop de Dakar.
} 
from attributes of contemporary society: materialism, greed, individualism, etc. Religion, as an instrument of alienation and its commodification, is at the origin of the horrible crime committed by Hermanas de Luz, followers of Saint Lucia. This article is a study based on a hermeneutic approach, reconciling the theoretical-conceptual and epistemological perspective with textual practice.

KEYWORDS: Religion. Alienation. Commodification. Reporting. Dehumanization.

\section{Introduction}

Quelque fulgurantes que puissent être les avancées de la science et de la technologie dans ce vingt unième siècle, force est de constater que l'homme continue, dans son vécu quotidien, d'être en butte à divers facteurs qui concourent tous à annihiler son épanouissement total. La survivance de ceux-ci, ainsi que leur caractère recrudescent, avaient d'ailleurs motivé l'ire de Roger Garaudy (1979) contre tout être humain qui, refusant de croire et d'œuvrer pour un futur meilleur, se laisserait gagner par une résignation qui, du coup, mettrait tout l'univers en péril ${ }^{1}$. Bien que cultivant un genre différent, Juan Madrid ne manque pas non plus de souligner, et même de décrier, ces travers qui, comme des termites, s'attaquent au fondement de toute société : l'être humain. Ces facteurs bloquants, dans Malos tiempos de Juan Madrid, sont de nature variée et peuvent même être le fait de croyances supraterrestres. La religion, que Cicéron a eu à définir comme « Le fait de s'occuper d'une nature supérieure que l'on appelle divine et de lui rendre un culte ${ }^{2}$, est dans cette œuvre madridiste mise sur la sellette du fait de ses effets déshumanisants. Ce travail s'articulera autour de deux axes principaux ou parties. Le premier sera essentiellement de portée théorique et discriminatoire. Tout discours écrit ou oral est une séquence ordonnée de mots ou de concepts qui, au cours de leur histoire, ont pu subir des distorsions assez conséquentes (PANACCIO, 1991, p. 26-27). De ce fait, pour mener à bien notre réflexion et éliminer toute entrave au processus de transaction qui lie tout émetteur à son destinataire ${ }^{3}$, nous nous

\footnotetext{
${ }^{1}$ L'auteur affirme, à la page 10 de son livre, que celui-ci a été écrit pour : "Dire aux résignés assis à regarder le torrent qui déferle et à ceux qui se sont déjà laissé emporter par lui vers le gouffre: II est possible encore d'exister et de vivre. Il est possible encore d'endiguer le flot, d'en inverser le cours".

${ }^{2}$ Cicéron. De l'invention oratoire, II, 53: "Religio est, quae superioris cuiusdam naturae, quam divinam vocant, curam caerimoniamque affert" (apud GRONDIN, 2009, p. 66-73).

${ }^{3}$ La sémiotisation du monde implique un double processus: l'un, le processus de transformation, qui, partant d'un "monde à signifier", transforme celui-ci en "monde signifié" sous l'action d'un sujet; l'autre, le processus de transaction, qui fait de ce "monde signifié" un objet d'échange avec un autre sujet qui joue le rôle de destinataire. Ce deuxième processus ne peut se réaliser que si les sujets, qui sont
} 
préoccuperons d'abord de définir le concept de religion en nous intéressant à son étymologie et à son évolution sémantique. Cet exposé préliminaire nous permettra d'aborder la deuxième partie, relative à l'étude de la religion dans Malos tiempos de Juan Madrid.

\section{Une approche sémantico-conceptuelle du vocable «religion»}

Ce n'est point chose aisée de vouloir définir la religion. Le terme renvoie à une expérience qui dépasse les concepts humains (MEN, 1991, p. 112-120) et sousentend une double diversité: une diversité du fait religieux lui-même aussi remarquable que son universalité et une diversité des théories du fait religieux, i.e., des acceptions distinctes selon les sujets (MARC, 1982, p. 98-100).

Selon la théorie cicéronienne, religion proviendrait du latin relegere, qui signifie relire. La religion était, en fait, une partie de la constitution romaine dont la préservation préoccupait au premier chef le natif d'Arpinum. La relecture avait pour objet les rites et les coutumes venus des ancêtres (CICÉRON, 1886, p. 53, livre II). C'était d'ailleurs cet acte qui distinguait fondamentalement le religieux du superstitieux qui passait des journées entières à adresser des prières aux dieux et à leur immoler des victimes pour que leurs enfants leur survécussent (CICÉRON, 1886, p. 155-56, livre II). L'homme "religieux", lui, repasse, choisit de relire ce qui concerne le culte divin. Cette approche cicéronienne sera jugée "inepte" par Lactance. Si la superstition et la religion ont le même objet, "qui est de rendre aux dieux le culte qui leur est dû", il serait incongru de vouloir opposer les deux termes:

Quelle raison m'apporterait-il pour montrer que ce soit un acte de religion de prier une fois les dieux pour la conservation de la vie des enfants, et que ce soit un acte de superstition de prier dix fois pour le même sujet? Si c'est bien fait de prier une fois pour obtenir cette grâce, c'est encore mieux fait de prier plusieurs fois. Si c'est bien fait de prier une heure, c'est encore mieux fait de prier tout le jour; si une victime apaise la colère des dieux et les rend favorables, plusieurs victimes les rendront encore plus favorables: des vœux redoublés plaisent plutôt qu'ils n'offensent; bien loin de haïr les serviteurs qui sont assidus à leur devoir, on les aime. Quelle raison y aurait-il de blâmer ceux qui chérissent plus tendrement leurs enfants que ne font les autres, et ceux qui rendent aux dieux des devoirs plus fréquents, et de louer ceux qui tiennent une conduite différente? On peut encore

impliqués, ont des univers de référence communs et partagent la même signification contenue dans l'acte de langage (CHARAUDEAU, 1995, p. 98-100). 
proposer le même argument de cette sorte: si c'est un crime d'employer tout le jour à faire des prières et à offrir des sacrifices, c'en est un d'y employer un seul moment; si c'est une superstition de demander souvent que vos enfants vous survivent, c'en est une de le demander rarement (LACTATIUS, Chap. XXVIII, Livre IV).

Lactance, s'insurgeant contre la folie de ses contemporains qui s'évertuent à vouloir chercher sur la terre l'objet de leur bonheur «au lieu de le chercher dans le ciel» (LACTANCIUS, Chap. Baillère I), faisait dériver la religion du latin religare (obstetricare: rattacher, relier). La religion, au lieu d'être une simple relecture de tout ce qui concerne le culte divin, vient du "[...] lien par lequel nous sommes attachés à Dieu: la piété nous tient comme liés à lui, et nous oblige de le servir comme notre maître et de lui obéir comme à notre père" (LACTANCIUS, Chap. XXVIII). Cette double étymologie sera commentée et discutée par nombre de Théologiens tels que Jean Calvin, entre autres. Leurs théories et observations retiendront l'une ou l'autre hypothèse, i.e., la religion comme lecture renouvelée ou la religion en tant que liaison plus étroite avec la Transcendance. Le réformateur Calvin pencha plutôt pour l'argument étymologique de Cicéron. Cependant, il donna au terme superstition une autre acception: celle de ne point se contenter des prescriptions divines, tout en gardant le couple oppositionnel. L'auteur de Institution de la religion chrestienne (CALVIN, 1859) résume en ces termes sa posture théologique:

Quant au mot de Religion, combien que Cicéron le déduise très bien du mot de Relire, toutesfois la raison qu'il ameine est forcée et prise de trop loin, c'est que les serviteurs de Dieu ont tousjours releu et diligemment médité ce qui estoit de faire. Or plustost j'estime que ce mot est opposé à la trop grande licence et excessive, que la pluspart du monde s'est permise, asçavoir de prendre à la volée tout ce qui luy venoit au-devant, mesme de voltiger légèrement çà et là. Religion donc emporte autant comme une retraite et discrétion meure et bien fondée, car la vraye piété, pour avoir certain arrest et ferme, se recueille en ses limites : comme il me semble que la Superstition a esté nommée, de ce qu'en ne se contentant pas de ce qui estoit ordonné de Dieu, elle a fait un amas superflu de choses vaines [...]. Or Dieu pour maintenir son droit, prononce qu'il est jaloux, et que si on le mesle parmi les dieux controuvez, il en fera rude vengence (CALVIN, 1959, p. 148).

Une telle polémique étymologique sera reprise par les ouvrages d'autorité des temps modernes. Le français Jean-Baptiste-Bonnaventure de Roquefort, dans son Dictionnaire étymologique de la langue française, paru au début du deuxième quart 
du $\mathrm{XIX}^{\mathrm{e}}$ siècle, donne au concept de religion une définition que les réflexions de Lactance arriment mieux. La religion serait: "culte rendu à la divinité; foi, croyance aux mystères, aux objets de ce culte; chose à laquelle on se croit indispensablement obligé. Du latin religio, dont la racine est ligare, lier, attacher, qui a formé religare, lier plus fortement" (DE ROQUEFORT, 1829, p. 312, t. 2). Une telle perception du fait religieux nous semble plus appropriée que celle de Lactance. Pour Georg Wilhelm Friedrich Hegel (1770-1831), les croyances africaines aux forces de la Nature ne s'identifient pas à un acte de foi, en quelque chose qui soit supérieur à l'homme (HEGEL, 1965, p. 253).

L'Africain se rend compte qu'elles [les forces de la nature] le dominent, mais pour lui ce sont des forces dont l'homme peut, d'une manière ou d'une autre, se rendre maître. II domine ces puissances naturelles. II ne faut pas penser ici à une adoration de Dieu ni à la reconnaissance d'un esprit universel opposé à celui de l'individu. L'homme ne connaît que lui-même, et lui-même comme opposé à la nature: c'est à cela que se réduit la rationalité chez ces peuples. Ils reconnaissent la force de la nature et cherchent à la dominer. C'est ainsi qu'ils croient que l'homme ne meurt jamais naturellement, mais que c'est la volonté d'un ennemi qui le tue par un pouvoir magique; pour empêcher cela, comme contre toute force naturelle, ils se servent à leur tour de la magie (HEGEL, 1965, p. 254).

Il ressort des propos du philosophe que si l'africain n'a pas idée de Dieu, c'est parce que cette idée est trop élevée pour que sa pensée puisse l'atteindre. L'africain entretient avec les forces de la nature une relation conflictuelle qui ne laisse même pas champ au sacré: ce rocher, cet arbre, cette source, ce caillou, cette pièce de bois, qui suscite en nous, en même temps, un sentiment de vénération, de respect et d'adoration (DURKHEIM, 1968, p. 51). Cependant, assujettir l'expérience religieuse à la seule existence d'un Être Suprême ne serait-il pas trop exclusiviste et n'aboutirait-il pas au déni hégélien de conscience religieuse chez le négro-africain ou tout autre peuple ne se retrouvant pas dans le référentiel du philosophe allemand?

\subsection{La religion, un terme globalisant et point discriminatoire}

Au nom d'un principe synthétique, un seul et unique terme est "[...] appliqué sans discrimination au Proche-Orient ancien, au judaïsme, au christianisme et à l'islam, ou à l'hindouisme, au bouddhisme et au confucianisme de même qu'aux 
peuples dits 'primitifs'" (ELIADE, 1970, p. 9). La religion, comme terme globalisant, ne peut "être utile" que s'il renvoie à la fois à une croyance en Dieu, en des dieux, en des esprits et à l'expérience du sacré. La hiérarchisation des religions théorisée par Hegel ne peut aucunement emporter notre adhésion, le postulat d'une religion supérieure appelée à surplanter toutes les autres ne peut-il dériver de l'affirmation implicite d'une race supérieure, destinée à diriger les autres? Selon ce philosophe allemand, la religion n'est pas un tout constitué, exempt des lois de l'évolution. Elle est un germe qui doit se développer, elle contient déjà en soi, d'une manière latente, "[...] tous les degrés de la future croissance et chacun de ces degrés, au moment où l'esprit y accède, se traduit en un type de religion déterminé" (VANCOURT, 1965, p. 115). Ainsi, les différentes formes de religions que sont I'hindouisme, le judaïsme et même l'islam ne représentent que "des moments de la religion en general". Elles "[...] constituent les degrés par où passe la notion de la religion pour que celle-ci parvienne à sa perfection. Cette religion révélée est la religion chrétienne" (HEGEL, 1959, p. 70. Le surlignage est notre).

Ce que Hegel appelle germe a existé sous sa forme la plus accomplie dans toutes les sociétés du monde. Aucune manifestation religieuse ne peut prétendre confiner les autres dans un état de complexe, en déterminant leur situation sur le mode de l'imperfection, de l'inauthenticité ${ }^{4}$. L'angoisse métaphysique s'est saisie de I'homme dès que celui-ci a vu ses besoins primaires satisfaits. Dans tous les coins du globe, des hommes et des femmes, de tout temps et de tout âge, ont toujours tenté de répondre aux questions fondamentales sur le sens de la vie: Qui sommesnous? D'où venons-nous? Où allons-nous? Cette recherche spirituelle s'est révélée très tôt dans l'histoire universelle de l'humanité.

Lorsque les hommes ne connaissaient pas encore "e secret de la fécondité humaine ni la relation entre le coït et l'enfantement" (STONE, 1979, p. 46), ils vénéraient la femme comme étant la seule et unique source de vie. À cette époque, dans presque toutes les régions du monde, les hommes et les femmes pratiquaient la religion de la déesse:

\footnotetext{
${ }^{4}$ Nous nous inspirons du célèbre passage de Sartre sur ce qu'il appelle -reprenant l'heureuse expression de Stekel- «le complexe judaïque». Sartre affirmait que: "[...] le juif se met en état de complexe lorsqu'il choisit de vivre sa situation sur le mode inauthentique. II s'est laissé persuader en somme par les antisémites, il est la première victime de leur propagande. II admet avec eux que, s'il y a un juif, il doit avoir les caractères que la malveillance populaire lui prête et son effort est pour se constituer en martyr, au sens propre du terme, c'est-à-dire, pour prouver par sa personne, qu'il n'y a pas de juif" (SARTRE, 1954. p. 114-115).
} 
Dans les sociétés du paléolithique supérieur, où la mère était considérée comme la seule et unique parente, où le culte des ancêtres constituait apparemment la base des rites sacrés et où la généalogie ne tenait compte que de la lignée des femmes, l'image que le clan se faisait du créateur de la vie humaine était celle de la toute première ancêtre, une femme qui fut alors déifiée comme l'Ancêtre Divine. Les nombreuses statuettes de femmes, appartenant aux cultures gravettienne et aurignacienne du paléolithique supérieur, nous fournissent la troisième série de preuves, la plus tangible. Certaines d'entre elles ont été datées du vingt-cinquième millénaire av. J.-C. Ces petites figures féminines, faites d'os, de pierre ou d'argile, ont été souvent appelées des Vénus. Elles ont été découvertes sur les emplacements d'anciennes petites communautés, le long de pans de murs affaissés, qui constituaient sans doute les tout premiers abris construits par des hommes sur cette terre (STONE, 1979, p. 48).

Ces mêmes statuettes représentant des divinités seront découvertes dans les communautés néolithiques qui surgissent avec les tout premiers signes de développement agricole (c'est d'ailleurs ce qui les définit comme néolithiques). Elles apparaissent dans la région appelée, plus tard, pays de Canaan (actuellement la Palestine, le Liban et la Syrie), en Anatolie (Turquie) et le long des rives de l'Euphrate et du Tigre (aujourd'hui l'Iraq et la Syrie) (STONE, 1979, p. 51). Au temps de la préhistoire, la divinité suprême de la Haute-Égypte (le Sud) était la Déesse Nekhebt, représentée par un vautour. En Basse-Égypte, (la région du delta), on adorait la Déesse sous la forme d'un cobra, appelé Ua Zit, le Grand Serpent. A partir du troisième millénaire avant notre ère, la Déesse, connue sous le nom de Nut, Net ou Nit (un dérivé de Nekhebt), était adorée comme le tout premier être, préexistant à toute créature (STONE, 1979, p. 78).

Le statut de père n'existant pas, la mère était donc le chef de famille, la seule responsable des générations futures. Les enfants prenaient le nom de la tribu ou du clan de leur mère. La descendance familiale s'effectuait selon la lignée des femmes, c'est-à-dire, de mère en fille, un système matrilinéaire qui prévaut jusqu'à présent chez certains peuples d'Australie où "[...] la filiation matrilinéaire et le mariage matrilocal (l'époux venant vivre dans la famille ou le village de sa femme) sont des coutumes généralisées, et le statut des femmes y est beaucoup plus élevé" (STONE, 1979, p. 46-47).

Ces religions antiques et non primitives, au sens où l'entendait Hegel, seront victimes de la masculinisation progressive des cultes du fait de la pratique de 
l'élevage qui exalte le rôle du mâle reproducteur ${ }^{5}$. Le rapport de l'homme avec le sacré se matérialisera alors sous d'autres formes: Animisme, Shintoïsme, Hindouisme, Bouddhisme, Confucianisme, Taoïsme, Mazdéisme, etc. À côté de ces expressions religieuses, qui se sont constituées à l'issue d'un ensemble d'interrogations, de réflexions et de recherches basées sur l'expérience acquise, il en existe d'autres communiquées par révélation divine: le Judaïsme, le Christianisme et I'Islam. Le processus de transaction ainsi établi nous servira de balise pour décrypter le traitement que Juan Madrid consacre à la religion dans son œuvre Malos tiempos.

\section{Malos tiempos, une chronique noire de notre époque}

Publié par les éditions Alianza Editorial en 2017, Malos tiempos réunit sous ce titre aussi évocateur huit récits qui à l'origine étaient des reportages publiés dans Cambio 16. Tournant le dos à toute vision euphorisante, "à l'eau de rose» de la société et de l'homme, ce sont tous des histoires de crime abjectes qui révèlent la face cachée des gens avec des personnages tirés du monde réel qui, comme dans le roman picaresque, parcourent tous les milieux et toutes les strates sociales. Le livre révèle le dessous des cartes et s'attribue une fonction spéculaire: montrer dans un miroir grossissant les travers de cette société qui se dit moderne et civilisée i.e. l'appétence foncière de certains producteurs ruraux, le matérialisme, la jalousie, l'égoïsme des classes nanties, etc. La religion sera aussi pointée du doigt non dans ses dogmes et/ou croyances fondamentales sinon de l'utilisation que les hommes en font.

\subsection{La religion: un instrument d'aliénation}

Le deuxième récit du livre, intitulé «La endemoniada», met en scène l'histoire de la petite Rosa Fernández Gonzálvez qui fut tuée atrocement par sa mère et les deux amies de celle-ci lors d'une séance d'exorcisme. La petite fille âgée de 11 ans fut la cible des excès meurtriers des pratiques cultuelles de sa génitrice, adepte de Sainte Lucie à laquelle le village tout entier vouait un culte: "Su madre se lo ténia dicho y su madre sabía mucho. Para eso, su madre era Hermana de Luz o Miradora

\footnotetext{
${ }^{5}$ Voir Vallet (2009).
} 
o Sanadora, que de estas tres maneras se dice. O sea, seguidora de la Hermana Lucía, Santa Lucía, que es esa santa que tiene aquí en el pueblo todo el mundo, que es una santa muy bendita que se arrancó los ojos" (MADRID, 2017, p. 48). Les Hermanas de luz du village de Almansa relèvent toutes d'une autorité qui tel un "directeur de conscience" (CODRON et al., 1971, p. 77$)^{6}$ prétendent les protéger et les guider:

Bueno pues las sanadoras que ya estaban tan cargadas de malo, tan llenas de maligno de tanto curar y curar que ya no podían más, pues iban a ver a don Enrique de Villena y este señor las curaba, les sacaba todos los malos que tenían en sus cuerpos.[...]. Parece que este señor es muy rico y muy misterioso y que es difícil verlo. [...] es el jefe de todas las sanadoras, hermanas de luz o lo que sea. El jefe espiritual, se entiende. Una especie de arzobispo, si valen las palabras (MADRID, 2017, p. 49).

Le culte de cette sainte était déjà très répandu au $\mathrm{V}^{\mathrm{e}}$, elle subit le martyre à Syracuse au IV ${ }^{\mathrm{e}}$ siècle et fut brulée vive ${ }^{7}$ pour la foi qu'elle professait vis-à-vis de l'église catholique. En fait, ce n'est point donc l'hagiographie de la sainte que le jeune narrateur met en doute mais plutôt le culte extrême qu'on lui voue dans le village au point d'en oublier complétement toute explication rationnelle ou logique des faits et autres événements qui ponctuent notre vie sur terre: "Según me decía a mí la gordita, su madre le contaba que la Hermana Lucía,o sea, Santa Lucía, es la que lo ve todo y la que tiene facultades y poderes, dados por Nuestro Señor Jesús, para sanar desgracias y apartar el malo, imponiendo las manos" (MADRID, 2017, p. 48). Tout dysfonctionnement physiologique (migraine, aménorrhée, etc.) était immédiatement lié au diable, Satan que seules les prières peuvent vaincre. La petite Rosa Fernandez Gonzálvez qui seulement souffrait d'un embonpoint prononcé fut littéralement étripée par sa propre mère et ses deux aides-soignantes qui croyaient fermement qu'elle était sous l'emprise du démon:

La sangre estaba por toda la habitación: por las paredes, por el suelo, por los muebles... sus vísceras y sus intestinos y todo lo que tenía dentro, debajo de la cama. También había por la habitación trocitos de carne, cristales rotos, una guitarra destrozada - la de mi

\footnotetext{
${ }^{6}$ Par ce terme, l'un des neuf prêtres dont les témoignages ont été recueillis dans le livre désigne les professeurs de son ancien séminaire, ces hommes qui savaient comment Dieu «appelle» et qu'il fallait suivre très docilement.

${ }^{7}$ Noël (1869). Ou encore une publication plus récente et qui a le mérite de réunir beaucoup d'autres hagiographies est celle de Cazal (2019).
} 
gordi, la que le habían regalado por Reyes -, una jofaina con orines y agua, donde nadaban fotografías rotas, platitos con aceite por todas partes, palomillas de aceite que daban luz y santos y santas por las paredes. Muchas estampitas de mucha gente bienaventurada pegadas en las paredes (MADRID, 2017, p. 64).

Une mort atroce qui, associée aux sévices corporelles que les Hermanas de Luz infligeaient à leurs proches, prend le contrepied de Pascal qui faisait de la religion le centre de toute chose:

Toute la conduite des choses doit avoir pour objet l'établissement et la grandeur de la religion [...], elle doit être tellement l'objet et le centre où toutes choses tendent, que qui en saura les principes puisse rendre raison et de toute la nature de l'homme en particulier, et de toute la conduite du monde en général (PASCAL, 1969, p. 49).

Juan Madrid, dans Malos tiempos, relate à quel point le fanatisme n'incite point la personne à exercer son libre arbitre, à augmenter son degré de conscience. L'évolution de la vie s'en trouve bloquée :

Cet immobilisme qui freine toute amélioration et toute adaptation à l'environnement est dangereux pour la survie de toute espèce et bien sûr pour la survie de l'homme. L'être qui n'évolue que de façon restreinte se met en danger: il risque de vivre de grandes épreuves, de souffrir et de régresser jusqu'à disparaître, dès l'instant où les conditions de vie se mettent à changer (MORANDO, 1998, p. 128).

Néanmoins "La endemoniada" ne revient pas seulement sur ce caractère pernicieux de certaines pratiques religieuses, le récit met aussi à nu un autre aspect non moins reluisant y afférent.

\subsection{La marchandisation de la religion}

La religiosité des Hermanas de Luz, le culte qu'elles vouent à Sainte Lucie ne sont point désintéressés. En échange des prières et autres onctions, les personnes qui concourent à leurs cabinets se doivent de faire des dons en espèces. Le narrateur souligne la subtilité de ces "entrepreneurs du sacré" qui refusant d'être payés, font appel cependant à la générosité des patients ou accompagnants à la suite de chaque séance: 
Doña Encarna, la sanadora, estuvo con la mano en mi barriga lo menos quince minutos sin abrir la boca ni decir nada. Luego terminó y le dijo a mi madre que me tuviera tres días a agua y a caldo de arroz, sin tomar nada más. También le dijo que me rezara las oraciones debidas a la Hermana Lucía y a Nuestro Señor Jesús, el que lo puede todo. [...].

Me acuerdo que mi madre le preguntó:

- ¿Cuánto es, doña Encarna?

Y la sanadora respondió:

-Yo no cobro dinero. Es designio del Altísimo lo que hago. Pero si quiere dar la voluntad...

Mi madre soltó tres billetes de mil pesetas, se volvió a persignar (a mí se me olvido esa vez) y nos fuimos para casa (MADRID, 2017, p. 53).

La niche financière que représentent les consultations des Hermanas semblent d'ailleurs expliquer leur nombre considérable pour un petit village comme Almansa. Elles sont deux cents au total à s'adonner à cette activité avec des fortunes diverses. La notoriété dont jouissait la mère de la petite victime Rosita et les gains conséquents avaient même amené le mari à démissionner de son travail pour se consacrer à l'entreprise familiale de soins:

Ochoa nos llevaba la señora ganando buenos dineros, ayudado por su marido, el bueno de Jesús, él siempre tan apocadito, tan poca cosa, el pobre, que hasta dejó el trabajo en la fábrica de zapatos para atender el negocio domiciliario. El bueno de don Jesús era el encargado de dar la vez y de hacer recados, con su carita de poca cosa que tiene el pobre hombre (MADRID, 2017, p. 58).

La religion devient ainsi un quasi-système économique du fait du consumérisme spirituel et de la hiérarchisation des compétences et valeurs. Selon Lionel Obadia, cette relation clientéliste au surnaturel n'est en aucun cas un fait des temps modernes (2013, p. 99). La relation entre le magicien et son client ou spectateur a toujours été construite sur un modèle économique, les "biens de salut" furent monnayés au Moyen Âge avec la pratique des indulgences. C'est d'ailleurs pour cette raison, qu'Obadia préconisait "une approche de l'économie religieuse par son historicité" (2013, p. 103).

\section{Conclusion}

Aux dires d'Aristote: "[...] la différence entre le chroniqueur et le poète ne vient pas de ce que l'un s'exprime en vers et l'autre en prose (on pourrait mettre en vers 
l'œuvre d'Hérodote, ce ne serait pas moins une chronique en vers qu'en prose); mais la différence est que l'un dit ce qui a eu lieu, l'autre ce qui pourrait avoir lieu" (ARISTOTE, 1980, p. 65). Juan Madrid, dans Malos tiempos invite le lecteur à un genre nouveau n'excluant plus le res factae ${ }^{8}$ du domaine littéraire pour témoigner d'une réalité en prise directe avec un vécu social. L'exigence aléthique enjoint l'auteur à mettre en texte des faits divers sanglants qui semblent proclamer la primauté de la raison sur toute chose. Au banc des accusés, figure la religion ou plutôt l'usage que certains hommes en font. Après avoir enterré Dieu, on assiste aujourd'hui au retour du religieux dans toutes les sphères de la société et sous une forme déshumanisante et mercantile qui brouille les pistes et les esprits. En fait, "La endemoniada" interpelle le lecteur sur la légitimité des croyances qui ne font que desservir l'être humain.

\section{Références}

ARISTOTE. La Poétique. Paris: Seuil, 1980.

AUGÉ, M. Génie du paganisme. Paris: Gallimard, 1982.

CALVIN, J. Institution de la religion chrestienne. Paris: Librairie de Ch. Meyrueis et Cie, 1859.

CAZAL, F. Les proverbes et les saints en Espagne au Siècle d'Or: Un aspect méconnu de de la figure du saint dans le Vocabulario de refranes y frases proverbiales de Gonzalo Correas (1627).Paris: BoD Books on Demand, 2019.

CHARAUDEAU, P. Une analyse sémiolinguistique du discours. Langages, vol. $29, \mathrm{n}^{\circ}$ 117,1995 , p. 96-111. Disponible sur: https://www.persee.fr/doc/lgge_0458726x_1995_num_29_117_1708, vol. 29. Consulté le 10/07/2019.

CICÉRON, M.T. De natura deorum. Paris: Ancienne librairie Germaine Baillère et Cie, 1886.

CODRON, J. et al. Prêtres de quelle église? Paris: Édition du Seuil, 1971.

DE ROQUEFORT, J.-B. B. Dictionnaire étymologique de la langue française. Paris: Decourchant, 1829.

\footnotetext{
${ }^{8}$ Une telle typologie bipartite que nous tenons d'Aristote peut être résumée très sommairement en une opposition entre Fiction (res fictae) et Vérité (res factae) ou encore, entre Fictivité et Factualité. Le res factae est du domaine de l'histoire. "Si fingat, peccat in historiam; si non fingat, peccat in poesin" (Si vous inventez, vous pêchez contre l'histoire; si vous n'inventez pas, vous pêchez contre la poésie)" (LÜSEBRINK, 1989. p. 07).
} 
DURKHEIM, E. Les formes élémentaires de la vie religieuse. Paris: P.U.F., 1968.

ELIADE, M. La nostalgie des origines. Paris: Gallimard, 1970.

GARAUDY, R. Appel aux vivants. Paris: Seuil, 1979.

GRONDIN,J. La Philosophie de la religion. Paris: PUF, 2009.

HEGEL, G. W. F. Leçons sur la philosophie de la religion. Paris: Vrin, 1959.

HEGEL, G. W. F. La raison dans l'histoire. Introduction à la philosophie de l'histoire. Montréal: Collège Saint-Jean-Vianney, 1965.

PANACCIO, C. Les mots, les concepts et les choses. La sémantique de Guillaume d'Occam et le nominalisme d'aujourd'hui. Montréal: Bellarmin, 1991.

LACTANTIUS, L. C. F. Institutions divines. De la vraie sagesse et de la vraie religion. Chap. XXVIII, Livre IV. Disponible sur: http://archive.wikiwix.com//cache/?url=http://remacle.org/bloodwolf/église/lactance. Consulté le 02/08/2019.

LÜSEBRINK, H.-J. De l'incontournabilité de la fiction dans la connaissance historique. Questionnements théoriques à partir de romans historiques contemporains d'Alejo Carpentier, de Yambo Oualoguem et d'Ousmane Sembène. Neohelicon XVI, $\mathrm{n}^{\circ}$. 2, 1989, p. $07 . \quad$ Disponible sur: http://link.springer.com/article/10.1007/BF02029114. Consulté le 02/08/2019.

MADRID, J. Malos tiempos. Madrid: Alianza editorial, 2017.

MEN, A. Les sources de la religion. Paris: Desclée de Brouwer, 1991.

MORANDO, P. Ce que vous devez savoir pour vous protéger des sectes. Paris: E.P.M., 1998.

NOËL, A. Vie de sainte Lucie, vierge et martyre de Syracuse, suivie de l'histoire de son culte et de sa translation à Metz. Metz: Rousseaux-Pallez, imprimeur de l'Evêché, 1869.

OBADIA, L. La marchandisation de Dieu. L'économie religieuse. Paris: CNRS Éditions, 2013.

PASCAL, B. Pensées. Paris: Didier, 1969.

SARTRE, J. P. Réflexions sur la question juive. Paris: Gallimard, 1954.

STONE, M. Quand Dieu était femme. À la découverte de la Grande Déesse, source du pouvoir des femmes. Montréal: L'Étincelle, 1979. 
VALLET, O. La revanche des dieux mâles. Le Monde des Religions, n³3, 2009. Disponible sur: http://www.lemondedesreligions.fr/archives/2009/01/01/la-femmedans-les- religions,9698203.php. Consulté le 03/05/2019.

VANCOURT, R. La pensée religieuse de Hegel. Paris: P.U.F., 1965.

Recebido em 27/09/2019

Aceito em 15/11/2019

Publicado em 27/11/2019 